\title{
Analysis of Perceptions of Milk Consumption and Customer Service in the City of Riobamba, Province of Chimborazo
}

\section{Análisis de Percepciones del Consumo de la Leche y Servicio Al Cliente en la Ciudad de Riobamba, Provincia de Chimborazo}

\author{
O. Parada Gutiérrez ${ }^{1}$, J. Vasco ${ }^{2}$, and C. Delgado ${ }^{2}$
}

VII International Congress of Science, Technology, Entrepreneurship and Innovation (SECTEI 2020)

Corresponding Author:

O. Parada Gutiérrez

oparadag@ulvr.edu.ec

Published: 26 August 2021

Production and Hosting by

Knowledge E

(c) O. Parada Gutiérrez et al. This article is distributed under the terms of the Creative Commons Attribution License, which permits unrestricted use and redistribution provided that the original author and source are credited.
S OPEN ACCESS
1Docente Titular, Facultad de Ciencias Sociales y Derecho, Universidad Laica Vicente Rocafuerte, Guayaquil, Ecuador

${ }^{2}$ Facultad de Administración de Empresas, Escuela Superior Politécnica de Chimborazo, Riobamba, Ecuador

\section{Abstract}

The customer has become a source of strategic information about the quality of products and services. For this reason, the objective of this research was to analyze the level of service and mental processes of clients in making decisions to consume milk in the province of Chimborazo. Non-probabilistic sampling was used and data were collected from 50 clients and 75 representatives of small- and medium-sized companies in the city of Riobamba which participated in the Production Exhibition, Macají, Riobamba 2019. An electroencephalogram was used to determine whether the purchasing decisions were made at the unconscious level by reading brain waves. Three brands of milk that are sold locally were used. The study revealed that there were representative differences in stimulating people to different perceptions (visual, olfactory, gustatory) that influence the appreciation and purchasing decisions of milk. Similarly, it was found that there are decisions that the consumer makes unconsciously based on memories and past experiences that influence their present behavior at the time of consumption. The study also revealed the need to improve the level of service.

Keywords: neuromarketing, perception, strategies, service level.

\section{Resumen}

El cliente se ha convertido en una fuente de información estratégica sobre la calidad del producto y del servicio. Por este motivo, el objetivo del artículo científico fue analizar el nivel de servicio y los procesos mentales del cliente para la toma las decisiones de consumir leche en la provincia de Chimborazo. El estudio se realizó mediante un muestreo no probabilístico con informaciones de 50 clientes y 75 representantes de pequeñas y medianas empresas de la ciudad de Riobamba que participaron en la Exposición de Producción, Macají, Riobamba 2019. Se utilizó el electroencefalograma para determinar si las decisiones de compra se realizan a nivel inconsciente mediante la lectura de ondas cerebrales. Para ello se utilizaron tres marcas de leche que se comercializan en la localidad. El estudio reveló que existen diferencias representativas al estimular a las personas a diferentes percepciones (visual, olfativa, gustativa) que influyen en la apreciación y decisión de compra de la leche. De igual modo, se constató que existen decisiones que realiza el consumidor de manera inconsciente basada en los recuerdos y experiencias del pasado que influyen en su comportamiento presente al momento del consumo. Asimismo, el estudio reveló la necesidad de mejorar el nivel de servicio. 


\section{Introducción}

La capacidad que tienen los consumidores de tomar decisiones, racionalizar sus pensamientos de acuerdo a sus creencias, valores y cultura determinan que este pueda elegir la opción que mayores beneficios en cuanto al consumo de productos o servicios en un mercado, esta racionalidad se puede fundamentar en buena parte en la teoría microeconómica donde las preferencias del consumidor se pueden ajustar en función de sus ingresos y su utilidad.

Adam Smith, mencionaba el egoísmo del ser humano: 'la anarquía del egoísmo, es lo que hace que el panadero trabaje por su pan, y exista el herrero, y con ellos el tendero, todos buscando su propio beneficio' [1]. En un sistema de sobrevivencia que está inmerso en el inconsciente las personas buscan satisfacerse, se consume porque se necesita, por deseo, por placer, es parte de la naturaleza humana, sin consumo no se podrían cubrir las necesidades para el propio beneficio humano.

El estudio realizado también tiene sus bases en la Economía Conductual (Behavioral Economics) que refiere la combinación de la psicología y la economía con relación a lo que ocurre en los mercados en los que algunos de los agentes económicos tienen limitaciones y complicaciones humanas en el momento de la toma de decisiones. En Mullainathan y Thaler [2] y Schnellenbach y Schubert [3] de manera general se plantea que los seres humanos se desvían del modelo económico estándar.

La racionalidad limitada refleja las capacidades cognitivas limitadas que limitan la resolución de problemas humanos. La fuerza de voluntad limitada capta el hecho de que las personas a veces toman decisiones que no están en su interés a largo plazo. El egoísmo limitado incorpora el hecho reconfortante de que los seres humanos a menudo están dispuestos a sacrificar sus propios intereses para ayudar a otros.

Según Mayorga et al. [4]: 'La economía conductual es una teoría del refuerzo que permite explicar diferentes comportamientos tanto animales como humanos enmarcados en la conducta de elección, donde los organismos toman decisiones a partir de las condiciones ambientales que se encuentran establecidas en un momento dado' ( $p$. 34).

Del mismo modo, Kahneman [5] apunta que la intuición y el razonamiento son vías alternativas en el momento de resolver problemas. En este sentido, la intuición se asemeja a la percepción y se atribuye a la emoción un papel significativo para la toma de decisiones. Tal es el caso del rol del optimismo en la asunción de riesgos, en las predicciones del perjuicio, y el papel de la satisfacción e insatisfacción en los pronósticos de los acontecimientos que tienen lugar en el entorno donde se desarrollan las actividades. Ello determina una brecha entre el enfoque tradicional basado en las creencias y las preferencias y el enfoque psicológico en los análisis de la toma de decisiones. 
En estudios más recientes [6], manifiesta que:

Las decisiones económicas no estarían basadas en los análisis de costobeneficio de optimización que la teoría económica ha sustentado corrientemente, sino más bien, en una mezcla de tensiones emocionales y desiderativas, las cuales se retroalimentan a sí mismas, y buscan un equilibrio conjunto, o un punto optimizador conjunto, con restricciones. Por lo tanto, de ellos se infiere que las decisiones de consumo no dependen de la racionalidad, aparece la neurociencia que identifica el efecto de las cargas emocionales del consumidor en el proceso de decisión de compra.

La parte racional del cerebro analiza el costo beneficio del consumo, pero está condicionada de manera directa por las áreas inconscientes del sistema límbico. Bajo esta premisa, [7], manifiesta que: 'La neurociencia no debe ser considerada como una disciplina, sino que es el conjunto de ciencias cuyo sujeto de investigación es el sistema nervioso con particular interés en cómo la actividad del cerebro, se relaciona con la conducta y el aprendizaje'.

El comportamiento de las personas es el resultado de las experiencias, la actividad cerebral para el procesamiento de esta información y su abstracción de la realidad, esto determina su conducta en un contexto, el cerebro acumula la información del medio ambiente donde se desarrolla, analiza la información, la racionaliza y toma decisiones.

El estudio del encéfalo lleva a la neurociencia ampliar su operatividad generando un sinnúmero de aplicaciones enfocadas en áreas fundamentales de gestión y conducción de las organizaciones. Determinando que el análisis de los procesos mentales de un individuo determina su nivel de planeación, de emociones y de sobrevivencia en el lugar donde se encuentre. Asimismo, [8] refiere que la aplicación de métodos de neurociencia para analizar y comprender el comportamiento humano relacionado con los mercados y el intercambio de marketing ha ganado recientemente la atención de la investigación. El objetivo básico es orientar el diseño y la presentación de los productos para optimizarlos para que sean lo más compatibles posible con las preferencias de los consumidores.

En [9] se desarrolla un complemento a la teoría del cerebro Tri-uno, en donde expone la composición del cerebro humano, y la divide en la corteza orbito-frontal, también denominada Córtex, que es la parte más racional del cerebro, la cual se encuentra apenas encima de los ojos, y es la parte encargada del planeamiento y el control de los impulsos. El sistema límbico y el sistema reptiliano.

El Córtex determina las funciones ejecutivas del ser humano que se agrupan en componentes como la capacidad de planificar y ejecutar sus actividades, selección de conductas u organización de tiempos y procesos, flexibilidad cognitiva y toma de decisiones racionales. El sistema límbico es el área emocional del cerebro que 
determinan las experiencias a través de las emociones que tienen las personas, los dos sistemas interactúan y se relacionan todo el tiempo para la toma de decisiones afectando la conducta de manera conjunta y determinando su comportamiento.

Quiere decir que las decisiones de consumo de las personas no son racionales en su totalidad sino más bien un proceso cognitivo por reconocimiento y afectivo determinado por asociaciones de las experiencias vividas.

El cerebro reptiliano está formado por los ganglios basales, el tallo cerebral y el sistema reticular. Es la parte del cerebro más primaria, se desarrolló hace unos 500 millones de años. Se caracteriza por ser asiento de la inteligencia básica, llamada también inteligencia de las rutinas.

Del anterior análisis se puede observar que en la actualidad las empresas se han convertido en emocionales porque promueven la diversidad de pensamiento, desarrollan la gestión del conocimiento como principal herramienta de desarrollo organizacional, conocen su cultura organizacional, su proyección al futuro y son capaces de identificar e involucrar al talento humano en sus procesos gerenciales de toma de decisiones.

Este reinventarse empresarial constituye base fundamental para el estudio del neuromarketing y el código emocional del consumidor.

El marketing emocional es la búsqueda en el ámbito de toda empresa de una conexión sostenible que haga que los clientes se sientan tan valorados y bien cuidados que se desviarán de su camino para ser leales, cuyo objetivo es generar un estado de lealtad tanto de los clientes como de la empresa con el fin de crear una relación que vaya más allá de un intercambio comercial, se busca crear y fortalecer una relación emocional que asegure establecer sentimientos positivos para fidelizar a los clientes a cierta marca, y a la empresa a cuidar de las necesidades de los consumidores [10, 11].

La relación entre empresa y consumidor produce nuevas experiencias y emociones que pueden ser positivas o negativas, estas determinan las futuras decisiones de compra llevando a un individuo esta asociación a un nivel de satisfacción o no por un producto o servicio.

Los códigos emocionales que se generan son el resultado de la globalización y la interacción de culturas que cambian todos los días, que se expresan en manera proactiva, reactiva y retroactiva. Proactiva, porque los códigos personales generan cambios en la moda y las costumbres; reactiva, porque muchos solamente obedecen a estas bolas de nieve, y retroactiva, porque otros encuentran su explicación emocional en lo pasado, retornando a lo básico [12].

Por su parte [13] refiere que: El neuromarketing es una disciplina de avanzada que investiga y estudia los procesos cerebrales que explican la conducta y la toma de decisiones de las personas en los campos de acción del marketing tradicional: Inteligencia de mercado, diseño de productos y servicios, comunicaciones, precios, branding, posicionamiento, targeting, canales y ventas. En adición, [14] refieren su 
aplicación acertada en organizaciones sin fines de lucro para el establecimiento de estrategias encaminadas a elevar la satisfacción social.

No obstante, las aplicaciones más generalizadas del neuromarketing o la neurociencia del consumidor están centradas en los negocios y representan pasar de la comercialización de instrumentos específicos, como el enfoque de grupos, la observación, la investigación directa, la entrevista a una serie de instrumentos que registran tanto la actividad eléctrica del cerebro como la actividad metabólica [15].

Los nuevos procedimientos de neuroimágenes proporcionan un enfoque efectivo para estudiar el comportamiento del consumidor. En tal sentido se destacan un conjunto de instrumentos que sirven de soporte a los diferentes experimentos que pueden realizarse. Entre ellos se destaca la técnica de Electroencefalografía (EEG). La misma es uno de los principales instrumentos utilizados para examinar la actividad cerebral. La técnica EEG es práctica, versátil, asequible, portátil y no invasiva para realizar análisis repetitivos en tiempo real de interacciones cerebrales.

Estas actividades neuronales consisten en dos tipos: ritmos; $y$ actividades transitorias [16].

Del mismo modo [16] apunta además que los ritmos son medidas de actividades colectivas sinápticas, neuronales y axonales de los conjuntos neuronales, en tanto, la actividad EEG se caracteriza por separar las frecuencias en bandas, denotadas como delta, theta, ritmos alfa, beta, gamma y mu. Dichas bandas de frecuencia se han relacionado con reacciones afectivas. La banda theta en el centro del frente del cerebro refleja el procesamiento emocional cuando un consumidor mira un producto. El alfa, la banda en la corteza prefrontal diferencia entre las valencias emocionales positivas y negativas. La banda beta se correlaciona con las alteraciones durante la excitación afectiva. Finalmente, la banda gamma se asocia en gran medida con los efectos de excitación.

Por otro lado, las actividades transitorias o potenciales de campo replican los potenciales de acción de ciertas neuronas de manera similar a los picos. Estos picos se pueden reconocer por su posición, frecuencia, amplitud, forma, recurrencia y propiedades operativas. En virtud de la misma las muestras de EEG deben promediarse sobre muchas iteraciones para descubrir los picos y eliminar las fluctuaciones de ruido.

La investigación desarrollada resulta necesaria debido a un conjunto de insuficiencias asociadas al consumo de la leche. En correspondencia a informaciones obtenidas del informe inicial del proyecto de investigación, 'Sostenibilidad y competitividad de la cadena de suministros de la leche de ganado vacuno en la provincia de Chimborazo', de la Facultad de Administración de Empresas, Escuela de Ingeniería en Marketing, en la cadena de suministros de la leche de ganado vacuno predominan un conjunto de insuficiencias. Las mismas fueron constatadas a través de la investigación empírica, la 
dirección de trabajos de prácticas pre profesionales, trabajos de titulación de estudiantes de pregrado y postgrado, así como revisión de informes de balance del gobierno local y entrevistas con ganaderos locales. También mediante la utilización de técnicas participativas de trabajos en grupos con los representantes del Centro Agrícola de Riobamba. Entre las principales insuficiencias se destacan: Disminución del consumo de leche, estrategias de ventas empíricas, insuficientes estudios de mercado, insuficientes campañas publicitarias para estimular el consumo de leche en la población local.

Del mismo modo, el servicio al cliente desde la logística constituye otro de los aspectos que influyen en el consumo y satisfacción del mercado. En este contexto se el servicio al cliente es el conjunto de actividades interrelacionadas que ofrece un suministrador con el fin de que el cliente obtenga el producto en el momento y lugar adecuado y asegure un uso correcto del mismo; ese servicio al cliente es una potente herramienta de marketing $[17,18]$. Asimismo, $[19,20]$ también refieren en su conceptualización el término actividades, a cuyo contexto y tomando los parámetros más significativos expuestos por los mismos, se arriba a entender por servicio al cliente a la relación que permite definir y alcanzar las actividades organizacionales con la satisfacción de las necesidades del cliente; para que este transmita esa satisfacción con nuevos públicos y potencie la calidad en el servicio con miras a la excelencia de la prestación del mismo.

La logística enfocada 'hacia el cliente' como su propio concepto lo enuncia, está orientada a satisfacer la demanda de cada cliente en tiempo, lugar, con la calidad requerida y al menor costo posible; de hecho, es uno de los enfoques de dirección estratégica encaminado a elevar la competitividad de la empresa incrementando el nivel de servicio y disminuyendo los costos. La atención al cliente o simplemente servicio al cliente es la actividad que desempeña una empresa para relacionarse con sus consumidores.

El servicio al cliente tiene gran importancia por ser la actividad clave que regula a las restantes, y que, por lo general, se encuentra relacionada con los objetivos empresariales; al definir el nivel y el grado de respuesta que debe tener el sistema logístico. Por ello, el establecimiento de estos niveles va a afectar al costo de la logística (a mejor y mayor servicio, mayor costo), pudiéndose llegar a la situación de que, si el nivel exigido es muy alto o los servicios son muy particulares, las alternativas para proporcionar dichos servicios sean tan restringidas que los costos lleguen a ser excesivamente altos.

Esta situación en el contexto de las pymes comercializadoras de leche presenta algunas insuficiencias que inciden no solo en un bajo nivel de servicios sino también en bajos volumen de ventas que afectan los costos y el posicionamiento de los mismos en el mercado. 
En tal sentido la cultura de calidad, el enfoque al cliente aún no refiere el enfoque integrador que exige la realidad contemporánea [21-23]. Algunas buenas prácticas se han orientado al mejoramiento del sistema de control de inventarios para adecuar los mismos a la demanda del mercado sobre la base de sistemas informativos y la utilización de las nuevas tecnologías de la información y las comunicaciones TIC's [24-26].

Al respecto el control como función administrativa se apoya en el uso de indicadores que permitan monitorear el nivel de servicio al cliente y tomar decisiones oportunas con un enfoque proactivo. No obstante, esta práctica en la gerencia de las pymes que comercializan la leche no ha sido una práctica generalizada ni sistemática [27].

La situación problémica planteada anteriormente refleja la ausencia de estudios referidos a evaluar el nivel de servicios y la conducta del consumidor para orientar el proceso de comercialización de la leche de manera efectiva y eficaz. De ahí, que el objetivo de este artículo es evaluar el nivel de servicio y las percepciones de los consumidores de la leche determinando su nivel de atención y reflexión exponiendo al sujeto de prueba a un estímulo de carácter kinestésico, olfativo y gustativo con diferentes marcas de leche que se venden en la localidad.

\section{Materiales y Métodos}

En la investigación se utilizó el electroencefalograma para analizar los procesos mentales del sujeto, se determinó niveles de atención y calma mental mediante estímulos kinestésicos, se trabajó con marcas de leche consideradas las más importantes en el mercado local y nacional.

Los participantes del estudio (50 personas) fueron seleccionados mediante un muestreo no probabilístico durante la celebración de la Feria de Macají en la ciudad de Riobamba en el mes de abril de 2017, lugar donde se encontraron los pequeños, medianos y grandes productores de leche a nivel regional y nacional, contexto adecuado para realizar la investigación.

Los estímulos sensoriales a los que fueron expuestos los participantes fueron olfativos, gustativos, en donde se realizó un análisis de sus niveles de atención para determinar su nivel de reflexión o decisión inconsciente al presentar una publicidad del producto. Los productos analizados fueron Parmalat, Nutrileche, La Lechera y la Leche a Granel dispensadas en los mercados locales.

La hipótesis sobre la apreciación olfativa, gustativa y kinestésica sobre una u otra marca de leche fue el punto de partida y fue ser validada mediante el estadístico de prueba Chi-cuadrado y la prueba de $\mathrm{T}$ de Student para muestras relacionadas. 
La comprobación de la hipótesis partió del cálculo de la media, mediana y moda del total de las muestras tomadas con el electroencefalograma. Para ello se utilizó el software SPSS.

En cuanto al análisis del servicio al cliente se realizó el cálculo de los siguientes indicadores:

$$
\begin{aligned}
& \text { Nivel de servicio de pedidos (NSP) }=\frac{\text { Pedidos entregados al 100\% }}{\text { Total de pedidos }} \\
& \text { Cumplimiento del plazo de entrega (CPE) }=\sum_{i=1}^{N} \frac{\text { Entregas en tiempo }}{N},
\end{aligned}
$$

Nivel de atención a las reclamaciones $(N A R)=\frac{\text { Reclamaciones atendidas }}{\text { Total de reclamaciones realizadas }}$,

Nivel de aceptación del servicio (NAS) $=\frac{\text { Unidades fisicas sin reclamación }}{\text { Unidades fisicas entregadas }}$,

$$
\text { Nivel de servicios de unidades (NSU) }=\frac{\text { Unidades entregadas }}{\text { Unidades solicitadas }},
$$

Nivel de atención de pedidos (NAP) $=\frac{\text { Total de pedidos atendidos }}{\text { Total de pedidos }}$

Nivel de servicio general (NSP*CPE*NAR*NAS*NSU*NAP).

Los indicadores de las fórmulas (1) y (4), caracterizan la respuesta del sistema logístico en cuanto a cantidad de artículos a entregar, mientras que los restantes valoran la respuesta en cuanto a diversidad, por lo que estos últimos caracterizan la flexibilidad de dicho sistema.

El análisis del servicio al cliente se realizó con informaciones aportadas por

75 pymes comerciales (muestreo no probabilístico) de la ciudad de Riobamba que participaron de la Feria de Macají con relación a sus principales proveedores de leche. En los momentos de recolectar la información de campo fue preciso usar la entrevista.

\section{Resultados y Discusión}

Como premisa al análisis del comportamiento del consumidor, se realizó un análisis de la producción de leche en la provincia de Chimborazo y su destino final (consumo). Para ello se utilizaron 533 observaciones del año 2016 referidas a la producción (litros de leche) en sectores, caseríos o recintos y su consumo productivo (destino), según las estadísticas del Ministerio de Agricultura y Ganadería en la provincia. Los resultados fueron los siguientes: 


\section{Table 1}

Producción y consumo productivo de la leche.

\begin{tabular}{|c|c|c|c|c|c|}
\hline \multicolumn{6}{|c|}{ Producción y consumo de leche } \\
\hline & $\mathbf{N}$ & Mínim o & Máxim o & Media & $\begin{array}{l}\text { Desviación } \\
\text { estándar }\end{array}$ \\
\hline Producción & 533 & 0,00 & 24000 & 867,928 & 1945,96132 \\
\hline de leche (litros) & & & 00 & 7 & \\
\hline Consumo & 533 & 0,00 & 23520 & 654,447 & 1764,95562 \\
\hline Productivo & & & 00 & 9 & \\
\hline $\begin{array}{l}N \text { válido (por } \\
\text { lista) }\end{array}$ & 533 & & & & \\
\hline
\end{tabular}

Fuente: Tabla de salida del software SPSS.

Según la Tabla 1, la mayor parte de la producción está dirigida al consumo productivo de la leche. Se pudo constatar que dicho consumo se realiza por los talleres artesanales de producción de quesos, los centros de acopio y las pasteurizadoras.

En la investigación también se realizó un análisis de la correlación existente entre la producción de litros de leche y la cantidad de litros de leche entregadas a las pasteurizadoras.

\section{Table 2}

\section{Correlaciones}

\begin{tabular}{lllll} 
& & Producción & Pasteurizadoras \\
Produccion & Correlación de Pearson & 1 & $0,133^{* *}$ \\
& Sig. (bilateral) & & 0,002 \\
\cline { 2 - 3 } & $N$ & 533 & 527 \\
\hline Pasteurizado ras & Correlación de Pearson & $0,133^{* *}$ & 1 \\
& Sig. (bilateral) & 0,002 & 533
\end{tabular}

** La correlación es significativa en el nivel 0,01 ( 2 colas).

Fuente: Tabla de salida del software SPSS.

Según los resultados de la Tabla 2 el coeficiente de correlación de Pearson es 0,133 . Existe una baja correlación entre la producción total de leche y la cantidad que se destina directamente a las pasteurizadoras. Significa que $(0,133) 2=1,7689$ es la proporción de varianza compartida entre ambas variables analizadas.

En cuanto al estudio de las percepciones de consumo de la leche de vaca en las distintas marcas analizadas a partir del encefalograma se obtienen los resultados siguientes:

Para el cálculo de los indicadores del nivel de servicios fueron consideradas en un primer momento el sector de la pequeña, mediana empresa, que cuenta con 
Table 3

Análisis comparativo gustativo.

Media
Mediana
Moda
Desviación
estándar

\begin{tabular}{l|l} 
Parmalat & Lechera \\
51,49 & 55,53 \\
50 & 50 \\
50 & 50 \\
15,877 & 14,417
\end{tabular}

Nutrileche
55,74
50
$50 a$
20,509

Leche de balde

47,87

50

50

18,643

Fuente: Tabla de salida del software SPSS.

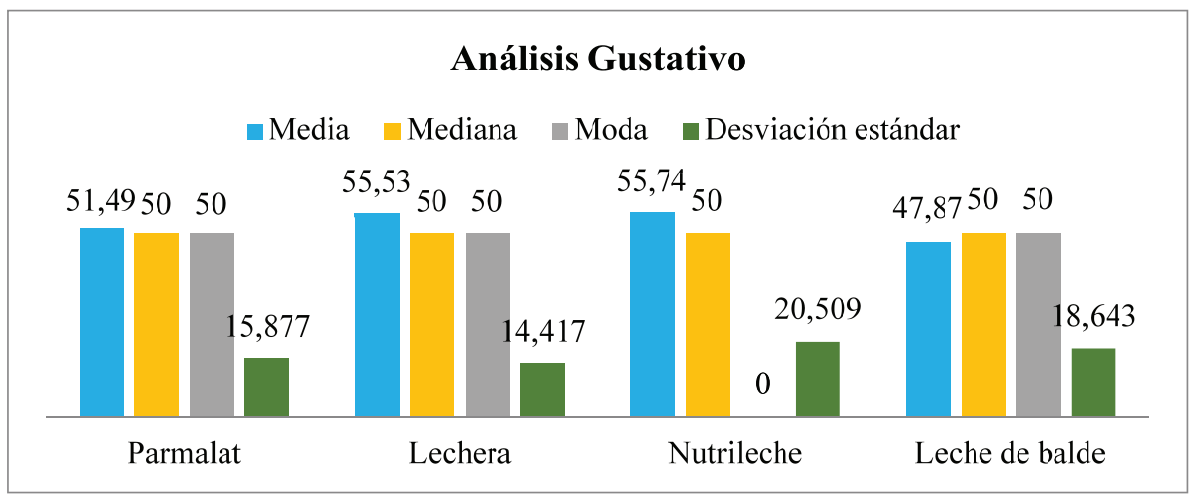

Figure 1

Análisis comparativo gustativo. Fuente: Encuestas.

Table 4

Análisis comparativo olfativo.

\begin{tabular}{lll} 
Análisis olfativo & \multicolumn{2}{l}{ Nutrileche } \\
\hline Media & Parmalat & 48,37 \\
Mediana & 50 & 50 \\
Moda & 60 & $40 a$ \\
Desviación & 19,004 & 16,875 \\
estándar & &
\end{tabular}

Lechera
51,22
50
40
18,779

Leche de balde
49,59
50
40
21,012

Fuente: Tabla de salida del software SPSS.

689 empresas según el censo económico del año 2010 realizado por el INEC. En esta parte del estudio se utilizó como muestra (75 empresas) en una primera etapa, que representan aproximadamente el $10 \%$ de las mismas (muestreo no probabilístico). Además, cuentan con proveedores de leche de marcas reconocidas en la ciudad.

La selección obedeció además a la disponibilidad de los empresarios de cooperar con la investigación, disponibilidad de tiempo, costos e información contable accesible para los investigadores. Es preciso destacar que este proceso es complejo debido al recelo que existe con la exposición de datos internos de los negocios, así como el nivel 


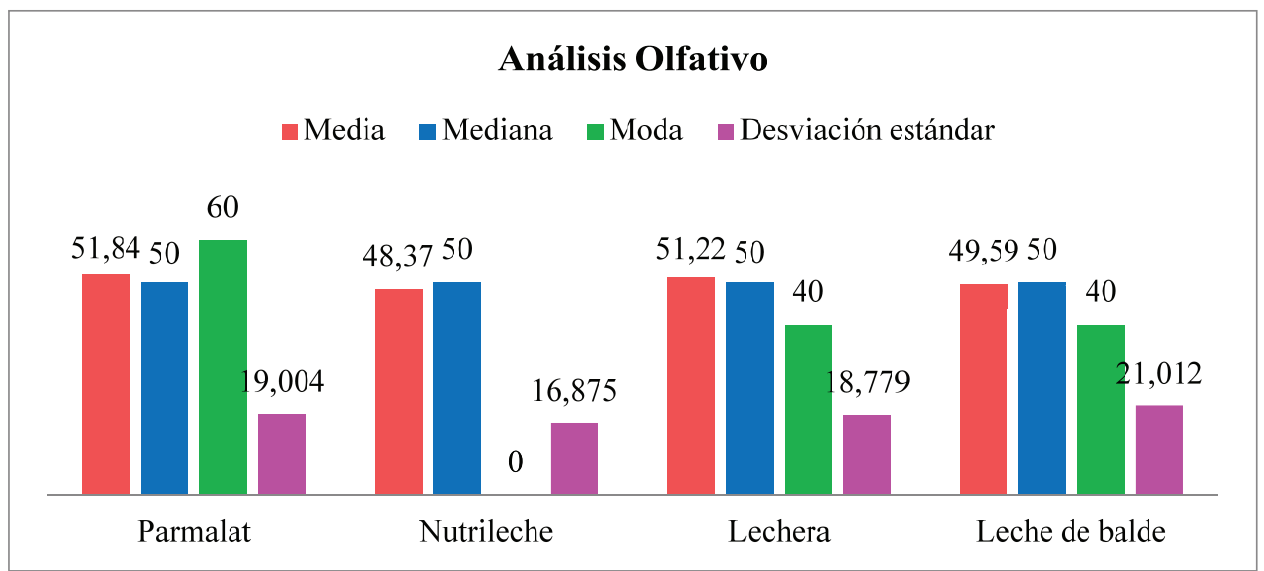

Figure 2

Análisis comparativo olfativo. Fuente: Encuestas.

Table 5

Análisis comparativo kinestésico.

\begin{tabular}{l|l|l|l} 
Análisis kinestésico & \multicolumn{2}{l}{ Nutrileche } & Lechera \\
\hline & Parmalat & 70 & 70,96 \\
\hline Media & 68,85 & 70 & 70 \\
\hline Mediana & 70 & 70 & 70 \\
\hline Deda & 80 & 16,568 & 17,741
\end{tabular}

Fuente: Tabla de salida del software SPSS.

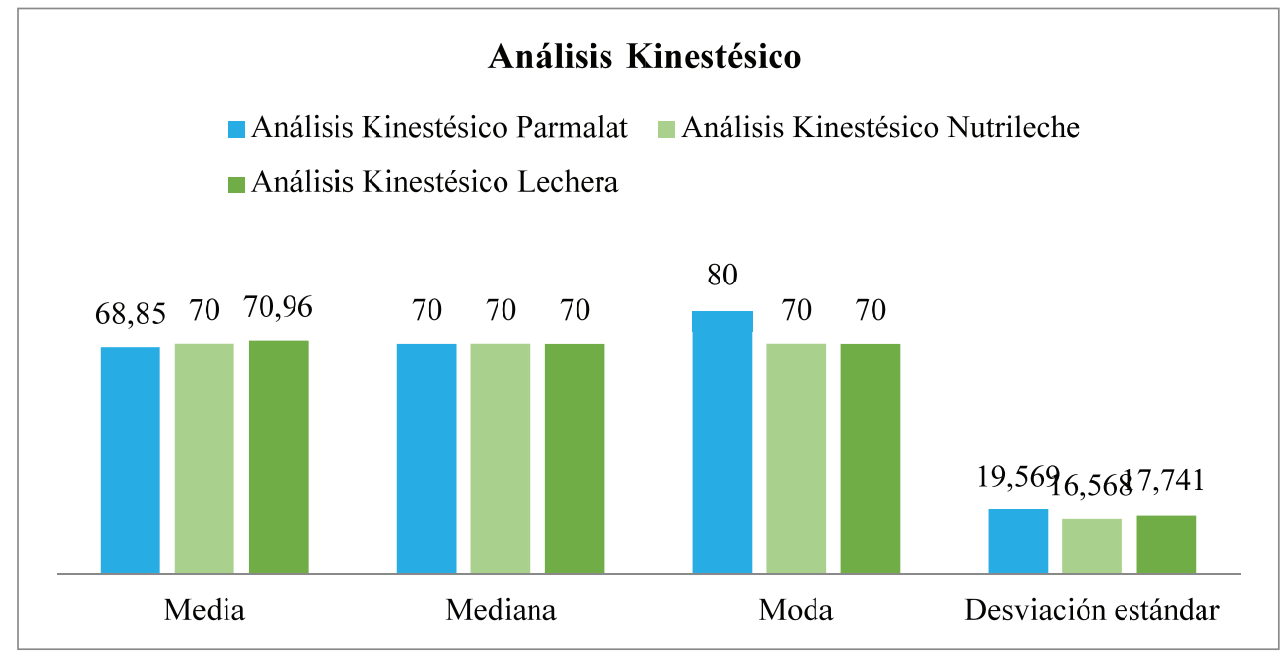

Figure 3

Análisis comparativo kinestésico. Fuente: Encuestas.

cultural de los representantes de las empresas. La información primaria para el cálculo 
Table 6

Prueba de muestras emparejadas método T-student

Diferencias emparejadas kinestésico

95\% de intervalo de confianza de la diferencia

\begin{tabular}{llllll} 
& Superior & $\mathbf{t}$ & gl & Sig. (bilateral) \\
$\begin{array}{l}\text { Par parmalat }-1 \\
\text { nutrileche }\end{array}$ & 5,464 & 3,873 & 27 & 0,001 \\
$\begin{array}{l}\text { Par parmalat - 2 } \\
\text { lechera }\end{array}$ & 2,293 & 1,8 & 27 & 0,083 \\
$\begin{array}{l}\text { Par nutrileche 3 - } \\
\text { lechera }\end{array}$ & $-0,79$ & -3 & 27 & 0,006 \\
\hline
\end{tabular}

Fuente: Tabla de salida del software SPSS.

de los indicadores fue resultado de la investigación de campo mediante entrevistas con los propietarios y/o administradores.

Con relación al cálculo de los indicadores de servicio al cliente se obtuvo los siguientes resultados:

\section{Table 7}

Resultados de indicadores de nivel de servicio.

\begin{tabular}{|c|c|c|c|c|c|c|c|}
\hline Proveedores de leche & NSP & CPE & NAR & NAS & NSU & NAP & Nsgral \\
\hline Carmen Carrillo - Prasol & 1 & 0,98 & 1,00 & 0,98 & 1,00 & 0,90 & 0,86 \\
\hline Cristina Silva - Avelina & 0,95 & 0,93 & 1,00 & 0,99 & 1,00 & 0,85 & 0,74 \\
\hline Paúl Noriega - Vita & 1,00 & 0,95 & 1,00 & 1,00 & 1,00 & 0,90 & 0,86 \\
\hline Iván Núñez - Parmalat & 1,00 & 0,95 & 1,00 & 1,00 & 1,00 & 0,95 & 0,90 \\
\hline $\begin{array}{l}\text { Francisco Vallejo - } \\
\text { Proalin }\end{array}$ & 0,96 & 0,95 & 1,00 & 0,95 & 1,00 & 0,95 & 0,82 \\
\hline Verónica Cisneros - Nutri & 0,98 & 0,95 & 1,00 & 1,00 & 1,00 & 0,95 & 0,88 \\
\hline $\begin{array}{l}\text { Luis Olmedo - Rey } \\
\text { Entera }\end{array}$ & 0,94 & 0,95 & 1,00 & 1,00 & 1,00 & 1,00 & 0,89 \\
\hline Toni Fábrica & 1,00 & 0,95 & 1,00 & 1,00 & 1,00 & 1,00 & 0,95 \\
\hline La Lechera Fábrica & 0,95 & 0,95 & 1,00 & 1,00 & 1,00 & 1,00 & 0,90 \\
\hline Pura Crema Fábrica & 0,95 & 0,95 & 1,00 & 1,00 & 1,00 & 1,00 & 0,90 \\
\hline \multicolumn{7}{|l|}{ PROMEDIO } & 0,87 \\
\hline
\end{tabular}

Fuente: Resultados de la investigación de campo.

\subsection{Discusión}

La mayor parte de la producción de leche en la provincia está dirigida al consumo productivo. Sin embargo, la entrega directa a las pasteurizadoras es baja con relación a otros destinos como es el caso de la producción de quesos y centros de acopio. En 
la investigación no se tuvo información precisa de la cantidad de leche que los centros de acopio proveen a las pasteurizadoras.

En cuanto al estudio gustativo de la leche, haciendo hincapié en la media aritmética, Nutrileche presenta mayor apreciación gustativa, ya que el valor bordea 55,74 mientras que la leche sin procesar o de balde es la que menos apreciación gustativa posee.

Según el análisis olfativo presenta mayor apreciación según la media aritmética la leche Parmalat con un valor de 51,84 a la muestra de 50 personas seguida de la Lechera, a continuación la leche sin procesar y finalmente Nutrileche con un valor de 48,37, el método estadístico para validar si existe o no una apreciación por una u otra leche fue Chi-cuadrado y McNemar dando un valor de 0,000.

En el análisis kinestésico se presentó una propaganda de 20 segundos para determinar los niveles de atención de anuncios publicitarios de las muestras de leche. Al respecto, la significancia según el método t de student para muestras relacionadas refiere que existe una apreciación por la publicidad entre la leche Parmalat y Nutrileche, de la misma manera existe una apreciación por la publicidad por las muestras de Nutrileche y La Lechera; pero no existe ninguna apreciación de la publicidad entre las leches Nutrileche y la Lechera.

Con relación al nivel de servicio general este se evalúa con un valor de 0,87, ello significa que los proveedores de leche a pymes comerciales de la ciudad de Riobamba satisfacen de manera general la demanda en un $87 \%$. Al respecto la investigación de campo reveló algunas falencias que afectan el nivel de servicio. Ellas son:

1. Insuficiente estudio de mercado, percepciones y expectativas de los consumidores de leche;

2. Baja calidad del pronóstico de la demanda de leche;

3. La negociación con los diferentes proveedores se desarrolla sobre base empíricas y la confianza es el requisito en que descansan los reaprovisionamientos;

4. No hay una política de inventarios fundamentada en el enfoque al cliente;

5. No hay estudios de preferencias de marcas, el precio continúa siendo la variable que decide el proceso de compra;

6. No existe una cadena de suministros estable y duradera en el tiempo.

El estudio reveló la necesidad de mejorar la calidad del servicio debido a la interrelación existente entre los elementos tangibles representados por el nivel de servicio logístico al cliente y la satisfacción de los clientes [28, 29]. Del mismo modo como apuntan [30, 31] es preciso desarrollar cadenas de suministros que permitan adoptar y evaluar múltiples estrategias para proteger el servicio al cliente en caso de que ocurran 
interrupciones que puedan conllevar a desabastecimientos y al costo social que ello representa.

\section{Conclusiones}

En la literatura consultada se aprecia una tendencia encaminada a caracterizar el neuromarketing como un campo emergente que vincula los lados cognitivo y afectivo del comportamiento del consumidor mediante el uso de la neurociencia. Sin embargo, su aplicación en el contexto empresarial es insuficiente a pesar del reconocimiento que se realiza acerca de su contribución al proceso de toma de decisiones con mayor nivel de certeza con relación al comportamiento del consumidor.

Aunque se han alcanzado progresos considerables las investigaciones sobre las evaluaciones neuronales pueden aumentar significativamente el poder predictivo más allá del de las evaluaciones tradicionales. Ello se debe a que las evaluaciones neuronales son mejores predictores que las evaluaciones autoinformadas y a la capacidad de los métodos de neurociencia para pronósticos en los que se destacan importantes herramientas como el electroencefalograma y las gafas para determinar eye tracking o seguimiento ocular.

En la investigación, los análisis conductuales básicos del comportamiento de consumo de leche realizados a partir de la aplicación del encefalograma, resultaron de gran utilidad práctica para para evaluar las percepciones de los consumidores desde las perspectivas olfativas y gustativas.

El estudio ha sido validado a través de informaciones obtenidas de 50 participantes de la Feria de Macají del 19 al 21 de abril de 2019. Para garantizar una mayor representatividad de los resultados, será necesario ampliar el tamaño de la muestra y estratificarla en correspondencia a los diferentes tipos de establecimientos comerciales que expiden leche en sus diferentes marcas. Sin embargo, los resultados refieren utilidad no sólo desde el punto de vista metodológico, sino también de utilidad práctica para mejorar el enfoque al cliente.

En el desarrollo y mejoramiento del nivel de servicio al cliente juega un papel importante la inclusión de indicadores y su fácil aplicación como forma de controlar el alcance de las acciones y la validación continua del procedimiento como herramienta útil en el mejoramiento del nivel de servicio al cliente.

\section{References}

[1] Adam S. La riqueza de las naciones. Madrid: Economía Alianza editorial; 1776. 
[2] Mullainathan S, Thaler RH. Behavioral economics. National Bureau of Economic Research. 2000;7948:100-119.

[3] Schnellenbach J, Schubert C. Behavioral political economy: A survey. European Journal of Political Economy. 2015;40:395-417.

[4] Mayorga P, Albañil N, Cómbita L. Elección y sustitución de estímulos entre el alcohol y el alimento en ratas: Una explicación del consumo de drogas desde el contexto de la economía conductual. Suma Psicológica. 2006;13:33-50.

[5] Kahneman D. Mapas de racionalidad limitada: Psicología para una economía conductual. Revista asturiana de economía. 2003;28:181-225.

[6] Glimcher P. Decisiones, incertidumbre y cerebro. México: Fondo de cultura económica; 2010.

[7] Kandel EJ. Neurociencia y conducta. Madrid: Prentice Hall; 1997.

[8] Khushaba RN, Wise C, Kodagoda S, Louviere J, Kahn BE, Townsend C. Consumer neuroscience: Assessing the brain response to marketing stimuli using electroencephalogram (EEG) and eye tracking. Expert Systems with Applications. 2013;40:3803-3812.

[9] MacLean P. The Triune brain evolution. New York: Plenun Press; 1990.

[10] Robinette SBC. Marketing emocional. New York: McGraw Hill; 2001.

[11] Eshuis J, Klijn EH, Braun E. Place marketing and citizen participation: branding as strategy to address the emotional dimension of policy making International. Review of administrative sciences. 2014;80:151-171.

[12] Cisneros A. Neuromarketing y economía. Bogotá: Ecoe Ediciones; 2012.

[13] Braidot N. Neuromarketing en acción. Buenos Aires: Granica; 2013.

[14] Flores J, Baruca A, Saldivar R. ¿ls neuromarketing ethical? Consumers say yes. consumers say no. Journal of Legal, Ethical and Regulatory Issues. 2014;17:77-91.

[15] Sebastian V. Neuromarketing and evaluation of cognitive and emotional responses of consumers to marketing stimuli. Procedia-Social and Behavioral Sciences. 2014;127:753-757.

[16] Khushaba RN, Wise C, Kodagoda S, Louviere J, Kahn BE, Townsend C. Consumer neuroscience: Assessing the brain response to marketing stimuli using electroencephalogram (EEG) and eye tracking. Expert Systems with Applications. 2013:40(9);3803-3812.

[17] Bobes AR, Valdés F, Pilar M. Servicio logístico al cliente en empresas de servicios: procedimiento para su diseño. Economía y Desarrollo. 2014;2:184-192.

[18] Campuzano NV, Ventura NVC. El servicio al cliente como filosofía y factor de posicionamiento de las cooperativas de taxis. Innova Research Journal. 2018:71-82.

[19] García A. Cultura de servicio en la optimización del servicio al cliente. Telos. 2016;18:381-398. 
[20] Villarraga DKO, Pedraza GAR. Sistemas de servicio al cliente implementados en las empresas: Un análisis de su eficiencia en el logro de la calidad del servicio. Innovar: Revista de ciencias administrativas y sociales. 2000; 143-152.

[21] Martínez R. Relación entre calidad y productividad en las PYMEs del sector servicios. Publicaciones en ciencias y tecnología. 2013;7(1):85-102.

[22] Gómez LC, Pérez EF. Propuesta de un modelo de gestión para PYMEs, centrado en la mejora continua. Síntesis tecnológica. 2017;3(2):59-67.

[23] Gavilánez MI, Espín Oleas ME, Palacios, MA. Impacto de la gestión administrativa en las PYMES del Ecuador. Observatorio de la Economía Latinoamericana. 2018 July. Disponible en: https://www.eumed.net/rev/oel/2018/07/ gestion-administrativa-pymes.html

[24] Gutiérrez OP, Rodríguez CAD, Obando CAS. Análisis de la oferta y merchandising en pymes comerciales. MktDescubre. 2016;1(08):137-145.

[25] Navarrete CV, Gutiérrez OP. Métodos para mejorar la eficiencia y la toma de decisiones en la gestión de inventarios. Revista Ciencia UNEMI. 2017;10(22): 2938.

[26] Singh D, Verma A. Inventory management in supply chain. Materials Today: Proceedings. 2018;5(2):3867- 3872.

[27] Cabeza MRQ, Quezada SCR, Gutiérrez OP. Procedimiento para incrementar la gestión competitiva de las pequeñas y medianas empresas (PYMES) comerciales ecuatorianas. Dominio de las Ciencias. 2017;3(4):364-383.

[28] Causado-Rodríguez E, Charris AN, Guerrero EA. Mejora continua del servicio al cliente mediante Servqual y red de Petri en un restaurante de Santa Marta, Colombia. Información tecnológica. 2019;30(2): 73-84.

[29] Gutiérrez G, Vásquez K, Vilca M. Influencia de la calidad de servicio en el posicionamiento de Plaza Vea Real Plaza Trujillo primer semestre 2018. SCIÉNDO. 2019;22(2):109-114.

[30] Schmitt AJ. Strategies for customer service level protection under multi- echelon supply chain disruption risk. Transportation Research Part B: Methodological. 2011;45(8):1266- 1283.

[31] Mazo AZ, Montoya RAG, Henao SAF. Indicadores logísticos en la cadena de suministro como apoyo al modelo SCOR. Clío América. 2014;8(15):90. 